\title{
Temporalising Tractable Description Logics
}

\author{
A. Artale \\ Faculty of Computer Science \\ Free University of Bozen-Bolzano \\ I-39100 Bozen-Bolzano, Italy \\ artale@inf.unibz.it \\ R. Kontchakov \\ School of CS and IS \\ Birkbeck College \\ London WC1E 7HX, U.K. \\ roman@dcs.bbk.ac.uk \\ C. Lutz \\ Department of Computer Science \\ Dresden University of Technology \\ 01062 Dresden, Germany \\ lutz@tcs.inf.tu-dresden.de
}

\author{
F. Wolter \\ Department of Computer Science \\ University of Liverpool \\ Liverpool L69 3BX, U.K. \\ frank@csc.liv.ac.uk
}

\author{
M. Zakharyaschev \\ School of Comp. Sci. and Inf. Syst. \\ Birkbeck College \\ London WC1E 7HX, U.K. \\ michael@dcs.bbk.ac.uk
}

\begin{abstract}
It is known that for temporal languages, such as firstorder $\mathcal{L} \mathcal{T} \mathcal{L}$, reasoning about constant (time-independent) relations is almost always undecidable. This applies to temporal description logics as well: constant binary relations together with general concept subsumptions in combinations of $\mathcal{L} \mathcal{T} \mathcal{L}$ and the basic description logic $\mathcal{A L C}$ cause undecidability. In this paper, we explore temporal extensions of two recently introduced families of 'weak' description logics known as $D L$-Lite and $\mathcal{E} \mathcal{L}$. Our results are twofold: temporalisations of even rather expressive variants of DL-Lite turn out to be decidable, while the temporalisation of $\mathcal{E} \mathcal{L}$ with general concept subsumptions and constant relations is undecidable.
\end{abstract}

\section{Introduction}

Over the last 15 years, many temporalised versions of description logics (DLs) have been suggested and investigated. We refer the reader to the survey papers and monograph $[6,14,4]$ where the history of the development of both interval and point-based temporal extensions of DLs is discussed in full detail. Our main concern in this paper are extensions of DLs by point-based temporal logics, in particular the standard linear time temporal logic $\mathcal{L} \mathcal{T} \mathcal{L}$ (see [13] and references therein). The current state of the art in this field can be summarised as follows: it is generally agreed that the semantics of combined temporal description logics should be based on the Cartesian products of the flow of time (the natural numbers $\mathbb{N}$ for $\mathcal{L} \mathcal{T} \mathcal{L}$ ) and the domains of the DL interpretations. Thus, a model for the combined language consists of a flow of snapshots that represent the domains of interest at various time points. This semantics corresponds to the semantics of first-order temporal logics (more precisely, to first-order temporal models with constant domains; varying and expanding domains have been considered as well in temporalised DLs, but they are not within the scope of this paper). In fact, the translation of standard DLs into first-order logic can be extended to a translation of temporalised DLs into first-order temporal logics. For this semantics, the expressivity and computational complexity of combinations of $\mathcal{L T} \mathcal{L}$ and DLs extending the standard Boolean DL $\mathcal{A L C}$ have been completely classified [14, 4]. Instead of trying to summarise all the available results here, we only point out one of the main insights from this investigation:

- combinations of $\mathcal{L} \mathcal{T} \mathcal{L}$ and $\mathcal{A L C}$, which allow general concept inclusions (GCIs) $C_{1} \sqsubseteq C_{2}$, are decidable (in fact, usually EXPSPACE-complete) if, and only if, the temporal operators are not applied to binary relations (roles) and, more generally, no constraints are imposed on the binary relations.

In other words, as long as one only wants to reason about the temporal behaviour of axioms (corresponding to closed formulas) and concepts (corresponding to unary predicates), the resulting combination is likely to be decidable; but as soon as the combination allows reasoning about the temporal behaviour of binary relations it becomes undecidable. This phenomenon is well understood and reflected in the definition of, e.g., the monodic fragments of first-order tempo- 
ral logics $[17,12]$. In particular, the undecidability results hold for the most important temporal constraint on binary relations, namely, that a role is constant over time: even a single constant role results in an undecidable combination of $\mathcal{A L C}$ and $\mathcal{L T} \mathcal{L}$ with GCIs. Without GCIs, temporal description logics may be decidable even with constant roles [14].

Unfortunately, many applications of temporal description logics (say, temporal data modelling, which will be briefly discussed in Section 3, or dynamic ontologies) require both GCIs and temporal constraints on roles, in particular constant roles. It was this problem that motivated the research which resulted in this paper. More precisely, our main aim was to find out whether it is possible to design useful combinations of $\mathcal{L} \mathcal{T} \mathcal{L}$ and DLs with GCIs and constant roles that are still decidable.

Recent developments in description logic have opened a new path to follow in designing such languages. First, the recognition of the importance of tractable reasoning and, in particular, query answering over DL ontologies with GCIs has given rise to the investigation of the new DL-Lite family of DLs [10, 11, 2]. And second, the use of huge DL-based ontologies with GCIs in bio- and medical informatics has led to the introduction and investigation of 'weak' DLs (reflecting the expressive power of existing ontologies) with tractable subsumption algorithms, namely, the $\mathcal{E} \mathcal{L}$-family of DLs $[5,7,8]$. Both families of DLs lack some of the expressive power of $\mathcal{A L C}$ but have nevertheless proved expressive enough for a number of applications. In this paper, we explore to which extent these new families of DLs can provide basis for useful and still decidable combinations of $\mathcal{L} \mathcal{T} \mathcal{L}$ and DLs with GCIs and constant roles.

The obtained results are twofold. On the one hand, we prove in Section 4 that the combination of one of the most expressive versions $D L$-Lite bool $_{\text {bo }}$ oL-Lite with $\mathcal{L} \mathcal{T} \mathcal{L}$ is indeed decidable (in EXPSPACE), even with GCIs and constant roles. Moreover, its Krom fragment turns out to be decidable in PSPACE. The proofs are based on an embedding into the one-variable fragment of first-order temporal logic. This means, in particular, that reasoning in temporal DL-Lite can be supported by available temporal provers; see, e.g., [12]. On the other hand, we show in Section 7 that the corresponding combination of $\mathcal{E} \mathcal{L}$ and $\mathcal{L T} \mathcal{L}$ is undecidable. The meaning of these results is analysed in Section 8.

\section{Temporal extension of DL-Lite bool}

We begin by introducing the temporal extension TDL-Lite bool $_{\text {of }}$ one of the most expressive description logics DL-Lite ${ }_{b o o l}$ of the DL-Lite family [2]. It combines the temporal operators of $\mathcal{L} \mathcal{T} \mathcal{L}, \bigcirc$ ('at the next moment') and $\mathcal{U}$ ('until'), with the language of DL-Lite ${ }_{b o o l}$ in a straightforward manner by applying them to concepts and Boolean combinations of GCIs and ABox assertions. Moreover, we will distinguish between local and global role names.

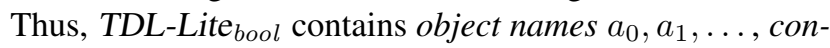
cept names $A_{0}, A_{1}, \ldots$, local role names $P_{0}, P_{1}, \ldots$, and global role names $T_{0}, T_{1}, \ldots$ Roles $R$, basic concepts $B$ and concepts $C$ of TDL-Lite ${ }_{b o o l}$ are defined as follows:

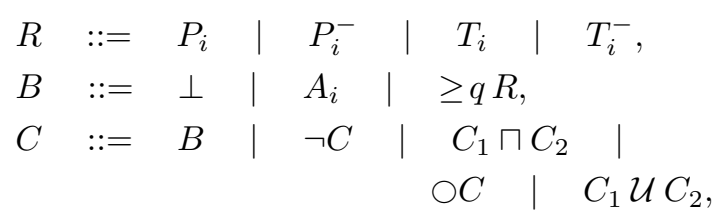

where $q \geq 1$ is a natural number (note that the results of this paper do not depend on whether $q$ is given in unary or

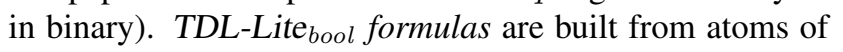
the form

$$
C_{1} \sqsubseteq C_{2}, \quad C\left(a_{i}\right), \quad R\left(a_{i}, a_{j}\right)
$$

with the help of the Boolean connectives (say, $\neg$ and $\wedge$ ) and the temporal operators $\bigcirc$ and $\mathcal{U}$. The atoms $C_{1} \sqsubseteq C_{2}$ are often called general concept inclusions (GCIs), while the atoms $C\left(a_{i}\right)$ and $R\left(a_{i}, a_{j}\right)$ are called ABox assertions.

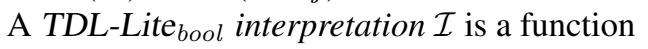

$\mathcal{I}(n)=\left(\Delta, a_{0}^{\mathcal{I}(n)}, \ldots, A_{0}^{\mathcal{I}(n)}, \ldots, P_{0}^{\mathcal{I}(n)}, \ldots, T_{0}^{\mathcal{I}(n)}, \ldots\right)$,

where $\Delta$ is a nonempty set, $n \in \mathbb{N}, a_{i}^{\mathcal{I}(n)} \in \Delta, A_{i}^{\mathcal{I}(n)} \subseteq \Delta$, $P_{i}^{\mathcal{I}(n)} \subseteq \Delta \times \Delta, T_{i}^{\mathcal{I}(n)} \subseteq \Delta \times \Delta$, with $a_{i}^{\mathcal{I}(n)}=a_{i}^{\overline{\mathcal{I}}(m)}$ and $T_{i}^{\mathcal{I}(n)}=T_{i}^{\mathcal{I}(m)}$, for all $n, m \in \mathbb{N}$, and $a_{i}^{\mathcal{I}(n)} \neq a_{j}^{\mathcal{I}(n)}$, for all $i \neq j$ and all $n \in \mathbb{N}$ (the last condition means the unique name assumption, which standard in DL). The role and concept formation constructors are interpreted in $\mathcal{I}$ as follows (where $R_{i}$ is either a local or global role name):

$$
\begin{aligned}
\left(R_{i}^{-}\right)^{\mathcal{I}(n)} & =\left\{(y, x) \mid(x, y) \in R_{i}^{\mathcal{I}(n)}\right\}, \\
\perp^{\mathcal{I}(n)} & =\emptyset, \\
(\geq q R)^{\mathcal{I}(n)} & =\left\{x \in \Delta \mid \sharp\left\{y \mid(x, y) \in R^{\mathcal{I}(n)}\right\} \geq q\right\}, \\
(\neg C)^{\mathcal{I}(n)} & =\Delta \backslash C^{\mathcal{I}(n)}, \\
\left(C_{1} \sqcap C_{2}\right)^{\mathcal{I}(n)} & =C_{1}^{\mathcal{I}(n)} \cap C_{2}^{\mathcal{I}(n)}, \\
(\bigcirc C)^{\mathcal{I}(n)} & =C^{\mathcal{I}(n+1)}, \\
\left(C_{1} \mathcal{U} C_{2}\right)^{\mathcal{I}(n)} & =\bigcup_{k>n}\left(C_{2}^{\mathcal{I}(k)} \cap \bigcap_{n<m<k} C_{1}^{\mathcal{I}(m)}\right) .
\end{aligned}
$$

The standard abbreviations $\top \equiv \neg \perp, \exists R \equiv(\geq 1 R)$, $C_{1} \sqcup C_{2} \equiv \neg\left(\neg C_{1} \sqcap \neg C_{2}\right), \leq q R \equiv \neg(\geq q+1 R)$, $(=q R) \equiv(\leq q R) \sqcap(\geq q R), \diamond_{F} C \equiv \top \mathcal{U} C$ ('some time in the future') and $\square_{F} C \equiv \neg \diamond_{F} \neg C$ ('always in the future') we need in what follows are self-explanatory and correspond to the intended semantics. 
The satisfaction relation $(\mathcal{I}, n) \models \varphi$, for a TDL-Lite bool formula $\varphi$, is defined inductively:

$$
\begin{aligned}
(\mathcal{I}, n) \models C_{1} \sqsubseteq C_{2} & \text { iff } C_{1}^{\mathcal{I}(n)} \subseteq C_{2}^{\mathcal{I}(n)}, \\
(\mathcal{I}, n) \models C\left(a_{i}\right) & \text { iff } a_{i}^{\mathcal{I}(n)} \in C^{\mathcal{I}(n)}, \\
(\mathcal{I}, n) \models R\left(a_{i}, a_{j}\right) & \text { iff }\left(a_{i}^{\mathcal{I}(n)}, a_{j}^{\mathcal{I}(n)}\right) \in R^{\mathcal{I}(n)}, \\
(\mathcal{I}, n) \models \neg \varphi & \text { iff }(\mathcal{I}, n) \not \varphi, \\
(\mathcal{I}, n) \models \varphi_{1} \wedge \varphi_{2} & \text { iff }(\mathcal{I}, n) \models \varphi_{1} \text { and }(\mathcal{I}, n) \models \varphi_{2}, \\
(\mathcal{I}, n) \models \bigcirc \varphi & \text { iff }(\mathcal{I}, n+1) \models \varphi, \\
(\mathcal{I}, n) \models \varphi_{1} \mathcal{U} \varphi_{2} & \text { iff there is } k>n \text { with }(\mathcal{I}, k) \models \varphi_{2} \\
& \text { and }(\mathcal{I}, m) \models \varphi_{1} \text { for all } n<m<k .
\end{aligned}
$$

We will also freely use the Booleans $\rightarrow$ and $\vee$ and the temporal operators $\square_{F}$ and $\diamond_{F}$ for formulas. A formula $\varphi$ is satisfiable if there is an interpretation $\mathcal{I}$ and a time point $n$ such that $(\mathcal{I}, n) \models \varphi$.

Observe that the interpretation of object names and global role names is time-independent, while the interpretation of local role names and concepts is allowed to vary over time. Time-independent concepts can be introduced by means of the axioms $\square_{F}^{+}(A \sqsubseteq \bigcirc A)$ and $\square_{F}^{+}(\bigcirc A \sqsubseteq A)$, where $\square_{F}^{+} \varphi \equiv \varphi \wedge \square_{F} \varphi$.

At first sight one might think that the satisfiability problem for this logic is undecidable because using a single global functional role $T$ (functionality can be ensured by the axiom $\geq 2 T \sqsubseteq \perp$ ) with functional $T^{-}$one can easily enforce the existence of a $\mathbb{N} \times \mathbb{N}$ grid, which could possibly be used to encode the undecidable $\mathbb{N} \times \mathbb{N}$ tiling problem. However, the language is not capable of expressing the requirements on colour matching in the domain 'dimension,' i.e., that if $(x, y) \in T^{\mathcal{I}(n)}$ then the colours of tiles covering $x$ and $y$ match (which can be easily expressed with the qualified existential quantifier $\exists T . C$ ). In fact, as we shall see in the next section, TDL-Lite one-variable fragment of first-order temporal logic, which is known to be decidable, actually, EXPSPACE-complete; see, e.g., [14]. Note that satisfiability in DL-Lite ${ }_{b o o l}$ is NPcomplete [2].

\section{Temporal data modelling with TDL-Lite ${ }_{b o o l}$}

Here we briefly discuss how TDL-Lite bool $_{\text {can }}$ be used for temporal data modelling. It was argued in [10] that the underlying DL DL-Lite bool $_{\text {can }}$ can represent atemporal conceptual data models like UML class diagrams and EntityRelationship models. For example, one maps entities $E$, denoting sets of abstract objects, into concept names $A_{E}$. Then one can represent the subclass relation (ISA) and disjointness between $E_{1}$ and $E_{2}$ by $A_{E_{1}} \sqsubseteq A_{E_{2}}$ and $A_{E_{1}} \sqsubseteq \neg A_{E_{2}}$, respectively, and to express that $E$ is covered by $E_{1}, \ldots, E_{n}$ one can use $A_{E} \sqsubseteq A_{E_{1}} \sqcup \cdots \sqcup A_{E_{n}}$ and $A_{E_{1}} \sqsubseteq A_{E}, \ldots, A_{E_{n}} \sqsubseteq A_{E}$. To capture an $n$-ary relationship $R$ over entities $E_{1}, \ldots, E_{n}$, one reifies the relationship. First, take a concept name $A_{R}$ and $n$ role names $R_{1}, \ldots, R_{n}$. The GCIs $A_{R} \sqsubseteq\left(=1 R_{i}\right)$ ensure that every instance of $A_{R}$ gives rise to a unique tuple in $R$; the GCIs $\exists R_{i}^{-} \sqsubseteq A_{E_{i}}$ guarantee that only instances of $E_{1}, \ldots, E_{n}$ may be connected by $R$. Participation constraints are captured by cardinality restrictions $A_{E_{i}} \sqsubseteq\left(\geq k R_{i}^{-}\right)$and $A_{E_{i}} \sqsubseteq\left(\leq m R_{i}^{-}\right)$. An attribute $P$ of an entity $E$, associating values of a concrete domain $D$ to instances of $E$, is considered as a binary relationship linking $E$ with $D$ : this can be captured by a concept $A_{P}$ and a pair of functional roles $P_{1}$ and $P_{2}$ with the GCIs $A_{P} \sqsubseteq\left(=1 P_{1}\right), A_{P} \sqsubseteq\left(=1 P_{2}\right)$, $\exists P_{1}^{-} \sqsubseteq A_{E}$ and $\exists P_{2}^{-} \sqsubseteq D$.

In the temporal context, we can express all those constraints using $\square_{F}^{+}\left(C_{1} \sqsubseteq C_{2}\right)$ instead of the atemporal $C_{1} \sqsubseteq C_{2}$. Below we write $C_{1} \sqsubseteq^{*} C_{2}$ for $\square_{F}^{+}\left(C_{1} \sqsubseteq C_{2}\right)$. However, even at this basic level, global roles are already required: when reifying relationships, to ensure that every instance of $A_{R}$ represents the same tuple at different times, the roles $R_{i}$ should be global; similarly, the roles $P_{1}$ and $P_{2}$ introduced for an attribute $P$ should be global. Moreover, concrete domains should be constant and disjoint: this is captured by $\left(D \sqsubseteq^{*} \bigcirc D\right) \wedge\left(\bigcirc D \sqsubseteq^{*} D\right)$, for all $D$, and $D \sqsubseteq^{*} \neg D^{\prime}$, for all distinct concrete domains $D, D^{\prime}$.

In addition, the temporal constructors of TDL-Lite bool are able to represent dynamic aspects of conceptual models. Timestamping is the basic temporal constraint used to model the temporal behaviour of entities, relationships and attributes [18, 3]. It is implemented either by marking entities, relationships and attributes as snapshot or temporary, or leaving them unmarked. An object belongs to a snapshot entity either never or at all times, no object may belong to a temporary entity at all times, and there are no temporal assumptions about instances of unmarked entities. The meaning of timestamps for relationships and attributes is analogous. In TDL-Lite bool timestamps are expressed by the following formulas: $\left(A_{E} \sqsubseteq^{*} \bigcirc A_{E}\right) \wedge\left(\bigcirc A_{E} \sqsubseteq^{*} A_{E}\right)$ for a snapshot/global entity and $\left(\top \sqsubseteq \diamond_{F}^{+} \neg A_{E}\right)$ for a temporary entity. Timestamping formulas for a relationship $R$ involve the concept name $A_{R}$ that reifies the relationship; then we need $\left(A_{R} \sqsubseteq^{*} \bigcirc A_{R}\right) \wedge\left(\bigcirc A_{R} \sqsubseteq^{*} A_{R}\right)$ for the snapshot/global relationship, and $\left(\top \sqsubseteq \diamond_{F}^{+} \neg A_{R}\right)$ for the temporary relationship. Attributes are treated similarly.

Finally, TDL-Lite ${ }_{b o o l}$ is capable of capturing dynamic transitions between entities where objects of a source entity, $E_{1}$, migrate to a target entity, $E_{2}$, with the help of the GCI $A_{E_{1}} \sqsubseteq^{*} \diamond_{F} A_{E_{2}}$.

It was observed in [1] that temporal conceptual models with timestamping and evolution constraints can be translated into the DL $\mathcal{D} \mathcal{L R}_{\mathcal{U S}}$ and that reasoning with temporal models with both timestamping and dynamic constraints is undecidable. The main difference here is that TDL-Lite bool 
lacks the ability to represent sub-relationships which is an essential part in the undecidability proof.

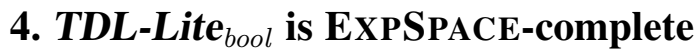

This result is proved by providing a satisfiability pre-

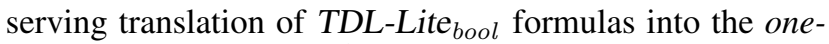
variable fragment $\mathcal{Q} \mathcal{T} \mathcal{L}^{1}$ of first-order temporal logic without function symbols and equality. To define the syntax of $\mathcal{Q T} \mathcal{L}^{1}$, fix one variable $x$. Then the formulas of $\mathcal{Q} \mathcal{T} \mathcal{L}^{1}$ are constructed from unary predicates $P(x)$ and $P\left(a_{i}\right)$ (where $a_{i}$ is a constant) and propositional variables $p$ using the standard connectives of first-order logic (with quantifiers $\forall x$ and $\exists x)$ and the temporal operators $\bigcirc$ and $\mathcal{U}$. $\mathcal{Q} \mathcal{T} \mathcal{L}^{1}$ models and the satisfaction relation between formulas and time points are defined in the obvious way by modifying the definition of TDL-Lite bool $_{\text {interpretations (however, there is }}$ no unique name assumption in this case); for details we refer the reader to [14], where the following is also shown:

Theorem 1. The satisfiability problem for $\mathcal{Q T} \mathcal{L}^{1}$-formulas is EXPSPACE-complete.

Now we define a translation ${ }^{\dagger}$ of TDL-Lite ${ }_{b o o l}$ formulas into $\mathcal{Q T} \mathcal{L}^{1}$. Let $\varphi$ be a TDL-Lite bool $_{\text {formula. De- }}$ note by role $(\varphi)$ the set of both local and global role names occurring in $\varphi$, by $g$-role $(\varphi)$ the set of global role names in $\varphi$, and by $o b(\varphi)$ the set of object names in $\varphi$. Let role $^{ \pm}(\varphi)=\left\{R, R^{-} \mid R \in\right.$ role $\left.(\varphi)\right\}$ and $g$-role ${ }^{ \pm}(\varphi)=$ $\left\{T, T^{-} \mid T \in\right.$ g-role $\left.(\varphi)\right\}$. Denote by $q_{\varphi}$ the maximum numerical parameter in $\varphi$.

With every object name $a_{i} \in o b(\varphi)$ we associate the individual constant $a_{i}$ of $\mathcal{Q T} \mathcal{L}^{1}$ and with every concept name $A_{i}$ the unary predicate $A_{i}(x)$ from the signature of $\mathcal{Q T} \mathcal{L}^{1}$. For each $R \in$ role $^{ \pm}(\varphi)$, we also introduce $q_{\varphi}$ fresh unary predicates $E_{q} R(x)$, for $1 \leq q \leq q_{\varphi}$. Intuitively, for each $n, E_{1} R(x)$ and $E_{1} R^{-}(x)$ represent the domain and range of $R$ at moment $n$ (i.e., $E_{1} R(x)$ and $E_{1} R^{-}(x)$ are interpreted by the sets of points with at least one $R$-successor and at least one $R$-predecessor at moment $n$, respectively), while $E_{q} R(x)$ and $E_{q} R^{-}(x)$ represent the sets of points with at least $q$ distinct $R$-successors and at least $q$ distinct $R$-predecessors at moment $n$. Additionally, for each pair $a_{i}, a_{j} \in o b(\varphi)$ and each role $R \in \operatorname{role}^{ \pm}(\varphi)$, we take a fresh propositional variable $R a_{i} a_{j}$ of $\mathcal{Q T} \mathcal{L}^{1}$ to encode $R\left(a_{i}, a_{j}\right)$.

By induction on the construction of a TDL-Lite bool $_{\text {con- }}$ cept $C$ we define the $\mathcal{Q T} \mathcal{L}^{1}$ - formula $C^{*}$ :

$$
\begin{aligned}
\perp^{*} & =\perp, & & \\
(A)^{*} & =A(x), & & (\geq q R)^{*}=E_{q} R(x), \\
(\neg C)^{*} & =\neg C^{*}(x), & & \left(C_{1} \sqcap C_{2}\right)^{*}=C_{1}^{*}(x) \wedge C_{2}^{*}(x), \\
(\bigcirc C)^{*} & =\bigcirc C^{*}(x), & \left(C_{1} \mathcal{U} C_{2}\right)^{*} & =C_{1}^{*}(x) \mathcal{U} C_{2}^{*}(x),
\end{aligned}
$$

where $A$ is a concept name and $R$ a role. Next, we extend this translation to TDL-Lite bool $_{\text {-formulas: }}$

$$
\begin{aligned}
& \left(C_{1} \sqsubseteq C_{2}\right)^{*}=\forall x\left(C_{1}^{*}(x) \rightarrow C_{2}^{*}(x)\right), \\
& \left(C\left(a_{i}\right)\right)^{*}=C^{*}\left(a_{i}\right), \quad\left(R\left(a_{i}, a_{j}\right)\right)^{*}=R a_{i} a_{j}, \\
& (\neg \psi)^{*}=\neg \psi^{*}, \quad\left(\psi_{1} \wedge \psi_{2}\right)^{*}=\psi_{1}^{*} \wedge \psi_{2}^{*}, \\
& (\bigcirc \psi)^{*}=\bigcirc \psi^{*}, \quad\left(\psi_{1} \mathcal{U} \psi_{2}\right)^{*}=\psi_{1}^{*} \mathcal{U} \psi_{2}^{*},
\end{aligned}
$$

where $C, C_{1}, C_{2}$ are concepts, $R$ is a role and $a_{i}, a_{j}$ are object names.

The following formulas express some natural properties of the role domains and ranges. For every $R \in \operatorname{role}^{ \pm}(\varphi)$, we need two $\mathcal{Q} \mathcal{T} \mathcal{L}^{1}$-sentences:

$$
\begin{aligned}
\varepsilon(R) & =\exists x E_{1} R(x) \rightarrow \exists x \operatorname{inv}\left(E_{1} R\right)(x), \\
\delta(R) & =\bigwedge_{q=1}^{q_{\varphi}-1} \forall x\left(E_{q+1} R(x) \rightarrow E_{q} R(x)\right),
\end{aligned}
$$

where $\operatorname{inv}\left(E_{1} R\right)$ is the predicate $E_{1} R_{k}^{-}$if $R=R_{k}$ and $E_{1} R_{k}$ if $R=R_{k}^{-}$. Sentence (1) says that if the domain of $R$ is not empty then its range is not empty either.

We also need formulas representing the relation of the $R a_{i} a_{j}$ with the unary predicates for the role domain and range. For a role $R \in \operatorname{role}^{ \pm}(\varphi)$, let

$$
\begin{aligned}
\omega(R) & =\bigwedge_{q=1}^{q_{\varphi}} \bigwedge_{\substack{a \in o b(\varphi) \\
a_{j_{1}}, \ldots, a_{j_{q} \in o b(\varphi)} \text { joj } j_{i} \neq j_{i^{\prime}} \text { for } i \neq i^{\prime}}}\left(\bigwedge_{i=1}^{q} R a a_{j_{i}} \rightarrow E_{q} R(a)\right) \\
\iota(R) & =\bigwedge_{a_{i}, a_{j} \in o b(\varphi)}\left(R a_{i} a_{j} \rightarrow \operatorname{inv}(R) a_{j} a_{i}\right)
\end{aligned}
$$

where $\operatorname{inv}(R) a_{j} a_{i}$ is the propositional variable $R_{k}^{-} a_{j} a_{i}$ if $R=R_{k}$ and $R_{k} a_{j} a_{i}$ if $R=R_{k}^{-}$.

For every global role $T \in g$-role ${ }^{ \pm}(\varphi)$ we need two additional sentences:

$$
\begin{aligned}
\gamma_{1}(T) & =\bigwedge_{q=1}^{q_{\varphi}} \forall x\left(E_{q} T(x) \leftrightarrow \bigcirc E_{q} T(x)\right), \\
\gamma_{2}(T) & =\bigwedge_{a_{i}, a_{j} \in o b(\varphi)}\left(T a_{i} a_{j} \leftrightarrow O T a_{i} a_{j}\right) .
\end{aligned}
$$

Finally, we set

$$
\begin{array}{r}
\varphi^{\dagger}=\varphi^{*} \wedge \bigwedge_{R \in \text { role }^{ \pm}(\varphi)} \square_{F}^{+}(\varepsilon(R) \wedge \delta(R) \wedge \omega(R) \wedge \iota(R)) \\
\wedge \bigwedge_{T \in g \text { ro }} \square_{F}^{+}\left(\gamma_{1}(T) \wedge \gamma_{2}(T)\right) .
\end{array}
$$

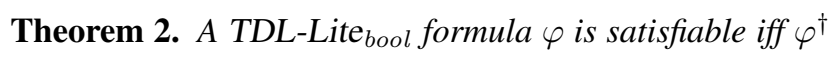
is satisfiable. 
Proof. $(\Leftarrow)$ Let $\mathfrak{M}$ be a first-order temporal model with a countable domain $D$ and let $(\mathfrak{M}, 0) \models \varphi^{\dagger}$. We denote the interpretation of unary predicates $P$ and propositional variables $p$ in $\mathfrak{M}$ at moment $n$ by $P^{\mathfrak{M}, n}$ and $p^{\mathfrak{M}, n}$. The interpretation of constants $a$ in $\mathfrak{M}$ is denoted by $a^{\mathfrak{M}}$. Let

$$
W_{0}=\left\{a^{\mathfrak{M}} \mid a \in o b(\varphi)\right\} \subseteq D .
$$

Without loss of generality we may assume that all the $a^{\mathfrak{M}}$ are distinct.

We are going to construct a TDL-Lite bool $_{\text {interpretation }}$ $\mathcal{I}$ satisfying $\varphi$ that is based on the domain

$$
\Delta=W_{0} \cup(D \times \mathbb{N})
$$

The interpretations of object names in $\mathcal{I}$ are given by their interpretations in $\mathfrak{M}: a^{\mathcal{I}(n)}=a^{\mathfrak{M}} \in W_{0}$. The interpretations $A^{\mathcal{I}(n)}$ of concept names $A$ in $\mathcal{I}$ are defined by taking

$$
A^{\mathcal{I}(n)}=\left\{w \in \Delta \mid(\mathfrak{M}, n) \models A^{*}[c p(w)]\right\},
$$

where the function $c p: \Delta \rightarrow D$ is defined as follows:

$$
c p(w)= \begin{cases}w, & \text { if } w \in W_{0}, \\ d, & \text { if } w=(d, n) \in D \times \mathbb{N} .\end{cases}
$$

We will call $w$ a copy of $c p(w)$. Now, for each $R \in \operatorname{role}(\varphi)$ and each $n \in \mathbb{N}$, we introduce inductively the interpretation $R^{\mathcal{I}(n)}$. (For global $R$ this can be done for some fixed $n$, say 0 , and then copied for all other $n$.) $R^{\mathcal{I}(n)}$ will be defined as the union

$$
R^{\mathcal{I}(n)}=\bigcup_{m=0}^{\infty} R^{n, m}
$$

where, for all $m \geq 0, R^{n, m} \subseteq W_{R}^{n, m} \times W_{R}^{n, m}$,

$$
W_{R}^{n, m} \subseteq W_{R}^{n, m+1} \text { and } \bigcup_{m=0}^{\infty} W_{R}^{n, m}=\Delta .
$$

We start with $W_{R}^{n, 0}=W_{0}$. The set $W_{R}^{n, m} \backslash W_{R}^{n, m-1}$, for $m \geq 0$, will be denoted by $V_{R}^{n, m}$; for convenience, we let $W_{R}^{n,-1}=\emptyset$, so that $V_{R}^{n, 0}=W_{0}$.

First we define the required $R$-rank $r^{n}(R, d)$ of $d \in D$ at moment $n$ :

$$
r^{n}(R, d)=\left\{\begin{array}{cc}
0, & \text { if }(\mathfrak{M}, n)=\neg E_{1} R[d], \\
q, & \text { if }(\mathfrak{M}, n)=E_{q} R \wedge \neg E_{q+1} R[d], \\
& \text { for } 1 \leq q<q_{\varphi}, \\
q_{\varphi}, & \text { if }(\mathfrak{M}, n)=E_{q_{\varphi}} R[d] .
\end{array}\right.
$$

It follows from (2) that $r^{n}(R, d)$ is a function and that if $d \in D$ and $r^{n}(R, d)=q$ then $(\mathfrak{M}, n) \models E_{q^{\prime}} R[d]$ whenever $1 \leq q^{\prime} \leq q$, and $(\mathfrak{M}, n) \models \neg E_{q^{\prime}} R[d]$ for $q<q^{\prime} \leq q_{\varphi}$.
We also define the actual $R$-rank $r_{m}^{n}(R, w)$ of $w \in \Delta$ at moment $n$ and step $m$ by taking

$r_{m}^{n}(R, w)=\left\{\begin{array}{c}q, \text { if } w \in \geq q R^{n, m} \cdot \Delta \backslash \geq q+1 R^{n, m} \cdot \Delta, \\ \text { for } 0 \leq q<q_{\varphi}, \\ q_{\varphi}, \text { if } w \in \geq q_{\varphi} R^{n, m} \cdot \Delta,\end{array}\right.$

where $\geq q S . \Delta=\{x \in \Delta \mid \sharp\{y \mid(x, y) \in S\} \geq q\}$, for a binary relation $S$.

For the basis of induction we set

$$
R^{n, 0}=\left\{\left(a_{i}^{\mathfrak{M}}, a_{j}^{\mathfrak{M}}\right) \in W_{0} \times W_{0} \mid(\mathfrak{M}, n) \models R a_{i} a_{j}\right\}
$$

By (3) and (4), for both $R$ and $R^{-}$(where $R^{--}=R$ ) and all $w \in W_{0}$,

$$
r_{0}^{n}(R, w) \leq r^{n}(R, c p(w))
$$

Suppose that the $W_{R}^{n, m}$ and $R^{n, m}$ have already been defined for $m \geq 0$. If we had $r_{m}^{n}(R, w)=r^{n}(R, c p(w))$, for both $R$ and $R^{-}$and all $w \in W_{R}^{n, m}$, then the interpretation $R^{n, m}$ we need for $R^{\mathcal{I}(n)}$ would have been constructed. However, in general this is not the case because there may be some 'defects' in the sense that the actual rank of some points is smaller than the required rank. Consider the following two sets of defects in $R^{n, m}$ :

$$
\begin{aligned}
& \Lambda_{R}^{n, m}=\left\{w \in V_{R}^{n, m} \mid r_{m}^{n}(R, w)<r^{n}(R, c p(w))\right\} \\
& \Lambda_{R^{-}}^{n, m}=\left\{w \in V_{R}^{n, m} \mid r_{m}^{n}\left(R^{-}, w\right)<r^{n}\left(R^{-}, c p(w)\right)\right\}
\end{aligned}
$$

The purpose of, say, $\Lambda_{R}^{n, m}$ is to identify those 'defective' points $w \in V_{R}^{n, m}$ from which precisely $r^{n}(R, c p(w))$ distinct $R$-arrows should start (according to $\mathfrak{M}$ ), but some arrows are still missing (only $r_{m}^{n}(R, w)$ many arrows exist). To 'cure' these defects, we extend $W_{R}^{n, m}$ to $W_{R}^{n, m+1}$ and $R^{n, m}$ to $R^{n, m+1}$ according to the following rules:

$\left(\Lambda_{R}^{n, m}\right)$ Let $w \in \Lambda_{R}^{n, m}$. Denote $q=r^{n}(R, d)-r_{m}^{n}(R, w)$ and $d=c p(w)$. Then $(\mathfrak{M}, n) \models E_{q^{\prime}} R[d]$ for some $q^{\prime} \geq q>0$. By (2), $(\mathfrak{M}, n) \models E_{1} R[d]$ and, by (1), there is $d^{\prime} \in D$ such that $(\mathfrak{M}, n) \models E_{1} R^{-}\left[d^{\prime}\right]$. In this case we take $q$ fresh copies $w_{1}^{\prime}, \ldots, w_{q}^{\prime}$ of $d^{\prime}$, i.e., $w_{1}^{\prime}, \ldots, w_{q}^{\prime} \in\left(\left\{d^{\prime}\right\} \times \mathbb{N}\right) \backslash W_{R}^{n, m}$, add them to $W_{R}^{n, m+1}$ and add the pairs $\left(w, w_{i}^{\prime}\right), 1 \leq i \leq q$, to
$R^{n, m+1}$.

$\left(\Lambda_{R^{-}}^{n, m}\right)$ Let $w \in \Lambda_{R^{-}}^{n, m-}$. Denote $q=r^{n}\left(R^{-}, d\right)-r_{m}^{n}\left(R^{-}, w\right)$ and $d=c p(w)$. Then $(\mathfrak{M}, n) \models E_{q^{\prime}} R^{-}[d]$ for some $q^{\prime} \geq q>0$. By (2), $(\mathfrak{M}, n) \models E_{1} R^{-}[d]$ and, by (1), there is $d^{\prime} \in D$ with $(\mathfrak{M}, n) \models E_{1} R\left[d^{\prime}\right]$. In this case we take $q$ fresh copies $w_{1}^{\prime}, \ldots, w_{q}^{\prime}$ of $d^{\prime}$, i.e., $w_{1}^{\prime}, \ldots, w_{q}^{\prime} \in\left(\left\{d^{\prime}\right\} \times \mathbb{N}\right) \backslash W_{R}^{n, m}$, add them to $W_{R}^{n, m+1}$ and add the pairs $\left(w_{i}^{\prime}, w\right), 1 \leq i \leq q$, to
$R^{n, m+1}$. 
( $\Omega$ ) Finally, if all defects for $R$ in $W_{R}^{n, m}$ have already been cured we take, for every $d \in D$, a fresh copy $(d, l) \in(\{d\} \times \mathbb{N}) \backslash W_{R}^{n, m}$ with minimal $l$ and add it to $W_{R}^{n, m+1}$.

It should be clear that the rule $(\Omega)$ guarantees that $\bigcup_{m=0}^{\infty} W_{R}^{n, m}=\Delta$. Now we observe the following important property of the construction: for all $m_{0} \geq 0$ and $w \in V_{R}^{n, m_{0}}$,

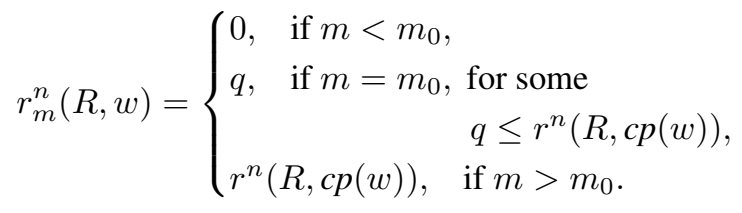

To prove this property, consider all possible cases. If $m<m_{0}$ then $w$ has not been added to $W_{R}^{n, m}$ yet, i.e., $w \notin W_{R}^{n, m}$, and so $r_{m}^{n}(R, w)=0$. If $m=m_{0}$ and $m_{0}=0$ then $r_{m}^{n}(R, w) \leq r^{n}(R, c p(w))$ follows from (10). If $m=m_{0}$ and $m_{0}>0$ then $w$ was added at step $m_{0}$ either to cure a defect of some point $w^{\prime} \in W_{R}^{n, m_{0}-1}$ or by $(\Omega)$. In the latter case we clearly have $r_{m}^{n}(R, w)=0$, and so $r_{m}^{n}(R, w) \leq r^{n}(R, c p(w))$. In the former case this means that either $\left(w^{\prime}, w\right) \in R^{n, m_{0}}$ and $w^{\prime} \in \Lambda_{R}^{n, m_{0}-1}$ or $\left(w, w^{\prime}\right) \in R^{n, m_{0}}$ and $w^{\prime} \in \Lambda_{R^{-}}^{n, m_{0}-1}$. In the first case

$$
(\mathfrak{M}, n) \models E_{1} R^{-}[c p(w)] .
$$

Since fresh witnesses $w$ are picked up every time the rule $\left(\Lambda_{R}^{n, m_{0}-1}\right)$ is applied and those witnesses satisfy (12), we obtain $r_{m_{0}}^{n}(R, w)=0, r_{m_{0}}^{n}\left(R^{-}, w\right)=1$ and $r^{n}\left(R^{-}, c p(w)\right) \geq 1$. The second case is similar. If $m=m_{0}+1$ then all defects of $w$ are cured at step $m_{0}+1$ by applying the rules $\left(\Lambda_{R}^{n, m_{0}}\right)$ and $\left(\Lambda_{R^{-}}^{n, m_{0}}\right)$. Therefore, $r_{m_{0}+1}^{n}(R, w)=r^{n}(R, c p(w))$. If $m>m_{0}+1$ then (11) follows from the observation that new arrows involving $w$ can only be added at step $m_{0}+1$, that is, for all $m \geq 0$,

$$
\begin{aligned}
R^{n, m+1} \backslash R^{n, m} & \subseteq \\
& \subseteq \\
V_{R}^{n, m} \times V_{R}^{n, m+1} & \cup V_{R}^{n, m+1} \times V_{R}^{n, m} .
\end{aligned}
$$

Finally, recall that if $R$ is global then, by (5) and (6), the above inductive procedure does not depend on $n$ and $R^{\mathcal{I}(n)}=R^{\mathcal{I}(m)}$, for all $n, m \in \mathbb{N}$.

It follows that, for all $R \in \operatorname{role}^{ \pm}(\varphi), 1 \leq q \leq q_{\varphi}, n \in \mathbb{N}$ and $w \in \Delta$,

$$
(\mathfrak{M}, n) \models E_{q} R[c p(w)] \quad \text { iff } \quad w \in \geq q R^{\mathcal{I}(n)} . \Delta .
$$

Indeed, if $(\mathfrak{M}, n) \models E_{q} R[c p(w)]$ then, by definition, $r^{n}(R, c p(w)) \geq q$. Let $w \in V_{R}^{n, m_{0}}$. Then, by (11), $r_{m}^{n}(R, w)=r^{n}(R, c p(w)) \geq q$, for all $m>m_{0}$. It follows from the definition of $r_{m}^{n}(R, w)$ and $R^{\mathcal{I}(n)}$ that $w \in \geq q R^{\mathcal{I}(n)} . \Delta$. Conversely, let $w \in \geq q R^{\mathcal{I}(n)} . \Delta$ and $w \in V_{R}^{n, m_{0}}$. Then, by (11), we have $q \leq r_{m}^{n}(R, w)=$ $r^{n}(R, c p(w))$, for all $m>m_{0}$. So, by the definition of $r^{n}(R, c p(w))$ and (2), we have $(\mathfrak{M}, n) \models E_{q} R[c p(w)]$.

Now we show by induction on the construction of concepts $C$ in $\varphi$ that, for all $n \in \mathbb{N}$ and $w \in \Delta$,

$$
(\mathfrak{M}, n) \models C^{*}[c p(w)] \quad \text { iff } \quad w \in C^{\mathcal{I}(n)} .
$$

The basis of induction is trivial for $B=\perp$ and follows from (7) if $B=A_{i}$ and (14) if $B=\geq q R$. The induction step for the Booleans $\left(C=\neg C_{1}\right.$ and $\left.C=C_{1} \sqcap C_{2}\right)$ and the temporal operators $\left(C=\bigcirc C_{1}\right.$ and $\left.C=C_{1} \mathcal{U} C_{2}\right)$ follows from the induction hypothesis.

Finally, we show that for each subformula $\psi$ of $\varphi$,

$$
(\mathfrak{M}, n) \models \psi^{*} \quad \text { iff } \quad(\mathcal{I}, n) \models \psi .
$$

For $\psi=C_{1} \sqsubseteq C_{2}$ and $\psi=C\left(a_{i}\right)$, this follows from (15). For $\psi=R_{k}\left(a_{i}, a_{j}\right),\left(a_{i}^{\mathcal{I}}, a_{j}^{\mathcal{I}}\right) \in R_{k}^{\mathcal{I}(n)}$ iff, by (13), $\left(a_{i}^{\mathcal{I}}, a_{j}^{\mathcal{I}}\right) \in R_{k}^{n, 0}$ iff, by (9), $(\mathfrak{M}, n) \models R_{k} a_{i} a_{j}$. The case $\psi=R_{k}^{-}\left(a_{i}, a_{j}\right)$ is similar. The induction step for the Booleans $\left(\psi=\neg \psi_{1}\right.$ and $\left.\psi=\psi_{1} \wedge \psi_{2}\right)$ and the temporal operators $\left(\psi=\bigcirc \psi_{1}\right.$ and $\left.\psi=\psi_{1} \mathcal{U} \psi_{2}\right)$ follows from the induction hypothesis.

Thus, we obtain $(\mathcal{I}, 0) \models \varphi$. The implication $(\Rightarrow)$ is straightforward.

The translation $\varphi^{\dagger}$ of $\varphi$ is obviously too lengthy to provide us with reasonably low complexity results. However, it follows from the proof above that in fact a lot of information in this translation is redundant and can be safely omitted. We define now a more concise translation of $\varphi$ into $\mathcal{Q T} \mathcal{L}^{1}$. For $R \in$ role $^{ \pm}(\varphi)$, let $Q_{\varphi}^{R}$ be the set of natural numbers containing 1 and all the numerical parameters $q$ for which $\geq q R$ occurs in $\varphi$. Then we set

$$
\begin{array}{r}
\varphi^{b}=\varphi^{*} \wedge \bigwedge_{R \in \text { role }} \square_{F}^{+}(\varphi) \\
\wedge \bigwedge_{T \in g \text {-role }}(\varepsilon) \wedge \square_{F}^{+}\left(\gamma^{b}(R) \wedge \omega^{b}(R) \wedge \iota(R)\right)
\end{array}
$$

where $\varepsilon(R), \iota(R)$ and $\gamma_{2}(T)$ are as before (see (1), (4) and (6), respectively),

$$
\begin{gathered}
\delta^{b}(R)=\bigwedge_{\substack{q, q^{\prime} \in Q_{\varphi}^{R}, q^{\prime}>q \\
q^{\prime}>q^{\prime \prime}>q \text { for no } q^{\prime \prime} \in Q_{\varphi}^{R}}} \forall x\left(E_{q^{\prime}} R(x) \rightarrow E_{q} R(x)\right), \\
\omega^{b}(R)=\bigwedge_{\substack { q \in Q_{\varphi}^{R} \\
\begin{subarray}{c}{a_{j_{1}}, \ldots, a_{j} \in o b(\varphi) \\
j_{i} \neq j_{i^{\prime}} \text { for } i \neq i^{\prime}{ q \in Q _ { \varphi } ^ { R } \\
\begin{subarray} { c } { a _ { j _ { 1 } } , \ldots , a _ { j } \in o b ( \varphi ) \\
j _ { i } \neq j _ { i ^ { \prime } } \text { for } i \neq i ^ { \prime } } }\end{subarray}} \bigwedge_{\substack{a \in o b(\varphi) \\
\gamma_{1}^{b}(T)}}\left(\bigwedge_{\substack{q \\
q \in Q_{\varphi}^{T}}} \forall x\left(E_{q} T(x) \leftrightarrow \bigcirc a_{j_{i}} \rightarrow E_{q} R(a)\right),\right.
\end{gathered}
$$


Corollary 3. A TDL-Lite ${ }_{b o o l}$ formula $\varphi$ is satisfiable iff the $\mathcal{Q T} \mathcal{L}^{1}$-sentence $\varphi^{b}$ is satisfiable.

Proof. Follows from the fact that $\varphi^{\dagger}$ is satisfiable iff $\varphi^{b}$ is satisfiable. Indeed, if $(\mathfrak{M}, 0) \models \varphi^{\dagger}$ then $(\mathfrak{M}, 0) \models \varphi^{b}$. Conversely, if $(\mathfrak{M}, 0) \models \varphi^{b}$ then one can construct a new model $\mathfrak{M}^{\prime}$ based on the same domain $D$ as $\mathfrak{M}$ by taking

- $A^{\mathfrak{M}^{\prime}, n}=A^{\mathfrak{M}, n}$, for all concept names $A$ and $n \in \mathbb{N}$;

- $E_{q} R^{\mathfrak{M}^{\prime}, n}=E_{q^{\prime}} R^{\mathfrak{M}, n}$, for $R \in$ role $^{ \pm}(\varphi), 1 \leq q \leq q_{\varphi}$ and $n \in \mathbb{N}$, where $q^{\prime}$ is the maximum number from $Q_{\varphi}^{R}$ with $q^{\prime} \leq q$;

- $R a_{i} a_{j}$ to be true in $\mathfrak{M}^{\prime}$ at $n$ iff $(\mathfrak{M}, n) \models R a_{i} a_{j}$, for all $R \in \operatorname{role}^{ \pm}(\varphi)$, all $a_{i}, a_{j} \in o b(\varphi)$ and all $n \in \mathbb{N}$;

- $a^{\mathfrak{M}^{\prime}}=a^{\mathfrak{M}}$, for all $a \in o b(\varphi)$.

It follows immediately from the definition that we have $\left(\mathfrak{M}^{\prime}, 0\right) \models \varphi^{\dagger}$. (For example, $\left(\mathfrak{M}^{\prime}, 0\right) \models \varphi^{*}$ follows from the fact that for every concept $(\geq q R)$ from $\varphi$ we have $E_{q} R^{\mathfrak{M}^{\prime}, n}=E_{q} R^{\mathfrak{M}, n}$, for all $n \in \mathbb{N}$.)

This observation makes it possible to prove the following result:

Theorem 4. The satisfiability problem for TDL-Lite ${ }_{b o o l}$ is EXPSPACE-complete.

Proof. As we know, satisfiability for $\mathcal{Q T} \mathcal{L}^{1}$ is EXPSPACEcomplete. However, we cannot use this result directly because the size of $\varphi^{b}$ is exponential in the number of object names (in fact, double exponential, if $q_{\varphi}$ is given in binary): $\left|\varphi^{b}\right| \leq$ const $\cdot|\varphi|+|o b(\varphi)|^{q_{\varphi}+1}$. Instead, we use the EXPSPACE algorithm presented in [14, Theorem 11.30] (see also [16]) which, given a $\mathcal{Q T} \mathcal{L}^{1}$-sentence $\psi$, decides whether $\psi$ is satisfiable or not by guessing an ultimately periodical quasimodel such that the lengths of its prefix and its period are bounded by some numbers $l_{1}$ and $l_{2}$, respectively. In general, both $l_{1}$ and $l_{2}$ are double exponential in the length $|\psi|$ of $\psi$. Hence, the algorithm requires single exponential space to write down the two numbers. The algorithm also requires exponential space to store at most 3 state candidates. Clearly, every realisable state candidate $\mathfrak{C}$ for $\varphi^{b}$ is uniquely determined by the following parameters:

- the set of propositional variables and the set of closed subformulas of the form $\forall x \chi(x)$ that belong to the types of $\mathfrak{C}$;

- for every type in $\mathfrak{C}$, the set of all open subformulas that belong to this type.

It is easy to compute that $\varphi^{b}$ contains $\mid$ role $\left.^{ \pm}(\varphi)|\cdot| o b(\varphi)\right|^{2}$ propositional variables and $|\varphi|+3 \cdot \mid$ role $^{ \pm}(\varphi) \mid$ closed subformulas of the form $\forall x \chi(x)$. Therefore, the 'propositional' part of a state candidate can be stored in space bounded by $p_{1}(|\varphi|)$, where $p_{1}$ is a polynomial. Next, for each type for $\varphi^{b}$, the number of open subformulas that belong to this type is bounded by $|\varphi|$, and the number of types in every state candidate is bounded by $2^{|\varphi|}$. Therefore, the 'type' part of a state candidate can be stored in space bounded by $2^{|\varphi|} \cdot|\varphi|$, and so the overall space required to store a state candidate for $\varphi^{b}$ is bounded by $2^{p_{2}(|\varphi|)}$, for some polynomial $p_{2}$. Now, [14, Theorem 11.26] provides more precise upper bounds on $l_{1}$ and $l_{2}$ :

$$
l_{1} \leq \sharp\left(\varphi^{b}\right) \quad \text { and } \quad l_{2} \leq k_{\varphi^{b}} \cdot \sharp\left(\varphi^{b}\right) \cdot b^{2}\left(\varphi^{b}\right)+\sharp\left(\varphi^{b}\right),
$$

where $\sharp\left(\varphi^{b}\right)$ is the number of distinct state candidates, $b\left(\varphi^{b}\right)$ the number of distinct types, and $k_{\varphi^{b}}$ the number of 'eventualities,' i.e., subformulas of $\varphi^{b}$ of the form $\chi_{1} \mathcal{U} \chi_{2}$. It follows from the above argument that $\sharp\left(\varphi^{b}\right) \leq 2^{2^{p_{2}(|\varphi|)}}$ and $b\left(\varphi^{b}\right) \leq 2^{p_{1}(|\varphi|)} \cdot 2^{|\varphi|}$ (every type for $\varphi^{b}$ is uniquely determined by its 'propositional' part and the subset of open subformulas that belong to it). Finally, the number $k_{\varphi^{b}}$ of 'eventualities' is bounded by $|\varphi|+2 \cdot \mid$ role $^{ \pm}(\varphi) \mid$. This shows that although the length of $\varphi^{b}$ is (double) exponential in $|\varphi|$, the numbers $l_{1}$ and $l_{2}$ are only double exponential in $|\varphi|$ (not triple exponential as one would expect). Therefore, the algorithm of [14, Theorem 11.30] runs in EXPSPACE.

The EXPSPACE lower bound follows from the fact that there is a satisfiability preserving polynomial translation from $\mathcal{Q T} \mathcal{L}^{1}$ to $T D L-$ Lite $_{\text {bool }}$. First, by introducing new unary predicates one can transform, in a satisfiability preserving way, each $\mathcal{Q T} \mathcal{L}^{1}$-formula into a $\mathcal{Q T} \mathcal{L}^{1}$-sentence containing neither $\exists x$ nor nested $\forall x$. Such a sentence $\varphi$ can be translated into TDL-Lite bool $_{\text {by }}$ by first associating with every unary predicate $P(x)$ a concept name $(P(x))^{\ddagger}=A_{P}$. For every subformula $\psi$ of $\varphi$ with free $x$, we obtain a concept $\psi^{\ddagger}$ by distributing the translation ${ }^{\ddagger}$ over the connectives $\bigcirc, \mathcal{U}, \neg$ and $\wedge$, e.g., $\left(\psi_{1} \wedge \psi_{2}\right)^{\ddagger}=\psi_{1}^{\ddagger} \sqcap \psi_{2}^{\ddagger}$. For each subformula of the form $\forall x \psi$, set $(\forall x \psi)^{\ddagger}=\left(\top \sqsubseteq \psi^{\ddagger}\right)$. Now, for $\mathcal{Q T} \mathcal{L}^{1}$-sentences, the translation.$^{\ddagger}$ again distributes over the connectives $\bigcirc, \mathcal{U}, \neg$ and $\wedge$. It is easily seen that $\varphi$ is satisfiable iff $\varphi^{\ddagger}$ is satisfiable.

The same lower bound follows also from Theorem 10 below.

\section{TDL-Lite krom $_{k \text { is PSPACE-complete }}$}

Consider now the Krom fragment TDL-Lite krom $_{\text {of }}$ TDL-Lite ${ }_{b o o l}$ with atomic formulas of the form

$$
\begin{gathered}
D_{1} \sqsubseteq D_{2}, \quad \neg D_{1} \sqsubseteq D_{2}, \quad D_{1} \sqsubseteq \neg D_{2}, \\
D\left(a_{i}\right), \quad R\left(a_{i}, a_{j}\right),
\end{gathered}
$$

where concepts $D_{1}, D_{2}$ are formed from basic concepts $B$ by means of $\bigcirc$ only:

$$
D::=B \mid O D .
$$


We can still apply all temporal operators and the Booleans to formulas. (Note that spatio-temporal logics of a similar kind were considered in [15] and [9]. Note also that satisfiability for the underlying DL DL-Lite $k_{k r o m}$ is NLOGSPACEcomplete [2].)

It is readily seen that the $\cdot{ }^{b}$-translations of TDL-Lite ${ }_{k r o m}$ formulas can be transformed in a satisfiability preserving way (by introducing abbreviations for nested $\bigcirc$ operators) to formulas of the following fragment $\mathcal{Q T} \mathcal{L}_{\text {krom }}^{1}$ of $\mathcal{Q T} \mathcal{L}^{1}$ :

$$
\begin{aligned}
Q(x) \quad:= & P_{i}(x) \quad \mid \quad \neg P_{i}(x), \\
L(x) \quad::= & Q(x) \quad \mid \quad O Q(x), \\
\varphi \quad::= & \forall x\left(L_{1}(x) \vee L_{2}(x)\right) \quad\left|\quad L\left(a_{j}\right) \quad\right| \\
& \neg \varphi\left|\varphi_{1} \wedge \varphi_{2}\right| \bigcirc \varphi \quad \mid \varphi_{1} \mathcal{U} \varphi_{2},
\end{aligned}
$$

where the $P_{i}$ are unary predicate symbols and the $a_{j}$ are constants. Predicates $P_{i}(x)$ and their negations $\neg P_{i}(x)$ will be called literals; literals $Q(x)$ and $\bigcirc$-prefixed literals will be called temporal literals.

In this section we establish (using the quasimodel technique from [14]) a PSPACE upper bound for satisfiability of $\mathcal{Q T} \mathcal{L}_{\text {krom }}^{1}$ formulas from which we obtain the following result (using Lemma 8 and an argument similar to the proof of Theorem 4):

Theorem 5. The satisfiability problem for TDL-Lite ${ }_{k r o m}$ formulas is PSPACE-complete.

We denote by $\dot{\neg} L(x)$ the formula equivalent to $\neg L(x)$ in the above restricted syntax, e.g., $\dot{\neg} \bigcirc P_{i}(x)$ is $\bigcirc \neg P_{i}(x)$ and $\dot{\neg} \neg P_{i}(x)$ is $\bigcirc P_{i}(x)$. For every formula of the form $\bigcirc Q(x)$, we reserve a unary predicate $Q^{\prime}(x)$ called the surrogate of $\bigcirc Q(x)$. Note that we introduce surrogates only for temporal literals (unlike 'standard' quasimodels, here we do not need to explicitly introduce surrogates for other temporal subformulas). Given a formula $\psi$, denote by $\bar{\psi}$ the result of replacing all subformulas of $\psi$ of the form $\bigcirc Q(x)$ by their surrogates.

For a $\mathcal{Q} \mathcal{T} \mathcal{L}_{\text {krom }}^{1}$ sentence $\varphi$, let $c l \varphi$ be the union of $\operatorname{sub}_{0} \varphi, \Sigma_{\varphi}$ and the $\Xi_{\varphi}^{a}$, for $a \in \operatorname{con} \varphi$, where $\operatorname{sub}_{0} \varphi$ is the set of closed subformulas of $\varphi, \operatorname{con} \varphi$ the set of all constants in $\varphi$, and

$$
\begin{aligned}
& \Lambda_{\varphi}=\left\{P_{i}(x), \neg P_{i}(x), \bigcirc P_{i}(x), \bigcirc \neg P_{i}(x) \mid\right. \\
& \left.P_{i}(x) \text { a predicate in } \varphi\right\}, \\
& \Sigma_{\varphi}=\left\{\forall x\left(L_{1}(x) \vee L_{2}(x)\right) \mid L_{1}(x), L_{2}(x) \in \Lambda_{\varphi}\right\}, \\
& \Xi_{\varphi}^{a}=\left\{L(a) \mid L(x) \in \Lambda_{\varphi}\right\}, \text { for } a \in \operatorname{con} \varphi .
\end{aligned}
$$

A state candidate $\mathfrak{C}$ for $\varphi$ is any subset of $c l \varphi$ satisfying the properties

$\left(\mathbf{q} \mathbf{s}_{K}^{0}\right) \chi_{\mathfrak{C}}=\bigwedge_{\psi \in \mathfrak{C} \cap \Sigma_{\varphi}} \bar{\psi}$ is satisfiable;

$\left(\mathbf{q} \mathbf{s}_{K}^{1}\right)$ for every $\psi \in \Sigma_{\varphi}$, if $\chi_{\mathfrak{C}} \models \bar{\psi}$ then $\psi \in \mathfrak{C}$; $\left(\mathbf{q} \mathbf{s}_{c}^{0}\right)$ for every $L(a) \in \Xi_{\varphi}^{a}, \quad \dot{\neg}(a) \in \mathfrak{C} \quad$ iff $L(a) \notin \mathfrak{C}$;

$\left(\mathbf{q} \mathbf{s}_{c}^{1}\right)$ for every $L_{1}(a), L_{2}(a) \in \Xi_{\varphi}^{a}$,

if $L_{1}(a), L_{2}(a) \in \mathfrak{C}$ then $\forall x\left(\dot{\neg} L_{1}(x) \vee \dot{\neg} L_{2}(x)\right) \notin \mathfrak{C}$;

(qs $\neg)$ for every $\neg \psi \in \operatorname{sub}_{0} \varphi, \quad \neg \psi \in \mathfrak{C}$ iff $\psi \notin \mathfrak{C}$;

$\left(\mathbf{q s} \mathbf{s}^{\wedge}\right)$ for every $\psi_{1} \wedge \psi_{2} \in \operatorname{sub} b_{0} \varphi$,

$$
\psi_{1} \wedge \psi_{2} \in \mathfrak{C} \text { iff } \psi_{1}, \psi_{2} \in \mathfrak{C} .
$$

Let $\boldsymbol{q}$ be a map associating with every $w \in \mathbb{N}$ a state candidate $\boldsymbol{q}(w)$ for $\varphi$. We call $\boldsymbol{q}$ a quasimodel for $\varphi$ if the following conditions hold:

$\left(\mathbf{q m}_{0}\right) \varphi \in \boldsymbol{q}\left(w_{0}\right)$, for some $w_{0} \geq 0$;

$\left.\mathbf{( q m}_{1}\right)$ for every $\forall x\left(\bigcirc Q_{1}(x) \vee \bigcirc Q_{2}(x)\right) \in \Sigma_{\varphi}$, $\forall x\left(\bigcirc Q_{1}(x) \vee \bigcirc Q_{2}(x)\right) \in \boldsymbol{q}(w)$

$$
\text { iff } \forall x\left(Q_{1}(x) \vee Q_{2}(x)\right) \in \boldsymbol{q}(w+1) ;
$$

$\left(\mathbf{q m} \mathbf{m}_{2}\right)$ for every $\bigcirc Q(a) \in \Xi_{\varphi}^{a}$,

$$
\bigcirc Q(a) \in \boldsymbol{q}(w) \text { iff } Q(a) \in \boldsymbol{q}(w+1) ;
$$

$\left(\mathbf{q m}_{3}\right)$ for every $\bigcirc \psi \in \operatorname{sub}_{0} \varphi$,

$$
\bigcirc \psi \in \boldsymbol{q}(w) \text { iff } \psi \in \boldsymbol{q}(w+1) ;
$$

$\left(\mathbf{q m}_{4}\right)$ for every $\psi_{1} \mathcal{U} \psi_{2} \in \operatorname{sub}_{0} \varphi, \quad \psi_{1} \mathcal{U} \psi_{2} \in \boldsymbol{q}(w)$ iff there is $k>0$ such that $\psi_{2} \in \boldsymbol{q}(w+k)$ and $\psi_{1} \in \boldsymbol{q}(w+n)$, for all $0<n<k$.

Lemma 6. $A \mathcal{Q} \mathcal{T} \mathcal{L}_{\text {krom }}^{1}$ sentence $\varphi$ is satisfiable iff there is a quasimodel for $\varphi$.

Proof. Suppose $\left(\mathfrak{M}, w_{0}\right) \models \varphi$. Then

$$
\boldsymbol{q}(w)=\{\psi \in \operatorname{cl} \varphi|(\mathfrak{M}, w)|=\psi\}
$$

defines a quasimodel for $\varphi$. Conversely, suppose that $\boldsymbol{q}$ is a quasimodel for $\varphi$.

Claim 7. If $\left\{L_{1}(x), \ldots, L_{k}(x)\right\} \subseteq \Lambda_{\varphi}$ and $\mathfrak{C}$ is a state candidate for $\varphi$, then

$$
\chi_{\mathfrak{C}} \wedge \quad \exists x\left(\overline{L_{1}}(x) \wedge \cdots \wedge \overline{L_{k}}(x)\right)
$$

is satisfiable iff there are no $1 \leq i, j \leq k$ such that $\forall x\left(\dot{\neg} L_{i}(x) \vee \dot{\neg} L_{j}(x)\right) \in \mathfrak{C}$.

Proof of claim. As formula (21) is a conjunction of the form $\forall x \chi_{1}(x) \wedge \exists x \chi_{2}(x)$, it is satisfiable iff the formula $\chi_{1}[a] \wedge \chi_{2}[a]$ is satisfiable, where $a$ is a constant symbol. Now, if $\chi_{1}[a] \wedge \chi_{2}[a]$ is satisfiable then there are no $i, j$ such that $\forall x\left(\neg L_{i}(x) \vee \dot{\neg} L_{j}(x)\right) \in \mathfrak{C}$. Conversely, suppose that there are no such $i, j$, but $\chi_{1}[a] \wedge \chi_{2}[a]$ is not satisfiable. Then $\chi_{1}[a] \models \neg \chi_{2}[a]$. By $\left(\mathbf{q s} \mathbf{s}_{K}^{0}\right), \chi_{1}[a]$ is satisfiable. Moreover as it is a $2-\mathrm{CNF}$,

$$
\chi_{1}[a] \models \neg \overline{L_{1}}[a] \vee \cdots \vee \neg \overline{L_{k}}[a]
$$

implies that there are $i, j$ with $\chi_{1}[a] \models \neg \overline{L_{i}}[a] \vee \neg \overline{L_{j}}[a]$. It follows from $\left(\mathbf{q s _ { K } ^ { 1 }}\right)$ that $\neg \overline{L_{i}}[a] \vee \neg \overline{L_{j}}[a]$ is a conjunct of $\chi_{1}[a]$, contrary to our assumption. 
Say that $\boldsymbol{t} \subseteq \Lambda_{\varphi}$ is a type for a state candidate $\mathfrak{C}$ if

- $L(x) \in \boldsymbol{t}$ iff $\dot{\neg} L(x) \notin \boldsymbol{t}$, for every $L(x) \in \Lambda_{\varphi}$;

- if $L_{1}(x), L_{2}(x) \in \boldsymbol{t}$ then $\forall x\left(\dot{\neg} L_{1}(x) \vee \dot{\neg} L_{2}(x)\right) \notin \mathfrak{C}$, for every $L_{1}(x), L_{2}(x) \in \Lambda_{\varphi}$.

By Claim 7, if $t$ is a type for $\mathfrak{C}$ then $\chi_{\mathfrak{C}} \wedge \exists x \wedge t$ is satisfiable. Denote by $T_{w}$ the set of all types for $\boldsymbol{q}(w)$. A pair of types $\left(\boldsymbol{t}, \boldsymbol{t}^{\prime}\right)$ is called suitable if $\bigcirc Q(x) \in \boldsymbol{t}$ iff $Q(x) \in \boldsymbol{t}^{\prime}$. Then the following two properties hold:

(succ) for each $\boldsymbol{t} \in T_{w}$ there is $\boldsymbol{t}^{\prime} \in T_{w+1}$ such that $\left(\boldsymbol{t}, \boldsymbol{t}^{\prime}\right)$ is a suitable pair;

(pred) for each $\boldsymbol{t}^{\prime} \in T_{w+1}$ there is $\boldsymbol{t} \in T_{w}$ such that $\left(\boldsymbol{t}, \boldsymbol{t}^{\prime}\right)$ is a suitable pair.

To show (succ), suppose that $t \in T_{w}$, but there is no $\boldsymbol{t}^{\prime} \in T_{w+1}$ such that $\left(\boldsymbol{t}, \boldsymbol{t}^{\prime}\right)$ is a suitable pair. Let $\bigcirc Q_{1}(x), \ldots, \bigcirc Q_{k}(x)$ be all temporal literals of the form $\bigcirc Q(x)$ in $\boldsymbol{t}$. Then

$$
\chi_{\boldsymbol{q}(w+1)} \wedge \exists x\left(Q_{1}(x) \wedge \cdots \wedge Q_{k}(x)\right)
$$

is not satisfiable. By Claim 7 , there are $i, j$ such that $\forall x\left(\dot{\neg} Q_{i}(x) \vee \dot{\neg} Q_{j}(x)\right) \in \boldsymbol{q}(w+1)$. Then, by $\left(\mathbf{q m}_{1}\right)$, $\forall x\left(\dot{\neg} \bigcirc Q_{i}(x) \vee \dot{\neg} \bigcirc Q_{j}(x)\right) \in \boldsymbol{q}(w)$, and so, by Claim 7 , the formula $\chi_{\boldsymbol{q}(w)} \wedge \exists x\left(\bigcirc Q_{1}(x) \wedge \cdots \wedge \bigcirc Q_{k}(x)\right)$ is not satisfiable, contrary to our assumption. Property (pred) is proved analogously.

Now we define a set $\mathfrak{R}$ of 'runs' through $\boldsymbol{q}$ by taking all

$$
r \in \prod_{w \in \mathbb{N}} T_{w}
$$

such that $(r(w), r(w+1))$ is a suitable pair for every $w$. By (succ) and (pred), for every $w$ and every type $\boldsymbol{t} \in T_{w}$ there is $r \in \mathfrak{R}$ such that $r(w)=\boldsymbol{t}$.

For $a \in \operatorname{con} \varphi$ and $w \in \mathbb{N}$, let

$$
\boldsymbol{t}_{a}^{w}=\left\{L(x) \in \Lambda_{\varphi} \mid L(a) \in \boldsymbol{q}(w)\right\} .
$$

It follows from $\left(\mathbf{q} \mathbf{s}_{c}^{0}\right)$ and $\left(\mathbf{q} \mathbf{s}_{c}^{1}\right)$ that the $\boldsymbol{t}_{a}^{w}$ are types. Moreover, by $\left(\mathbf{q m}_{2}\right),\left(\boldsymbol{t}_{a}^{w}, \boldsymbol{t}_{a}^{w+1}\right)$ is a suitable pair for every $w$. Thus, there is $r_{a} \in \mathfrak{R}$ with $r_{a}(w)=\boldsymbol{t}_{a}^{w}$, for every $w$.

Consider the model $\mathfrak{M}=\left(\mathfrak{R}, a_{0}^{\mathfrak{M}}, \ldots, P_{0}^{\mathfrak{M}, w}, \ldots\right)$, where $a_{j}^{\mathfrak{M}}=r_{a_{j}}$ and $P_{i}^{\mathfrak{M}, w}=\left\{r \in \mathfrak{R} \mid P_{i} \in r(w)\right\}$. It is readily checked that $\left(\mathfrak{M}, w_{0}\right) \models \varphi$.

Lemma 8. $A \mathcal{Q T} \mathcal{L}_{\text {krom }}^{1}$-sentence $\varphi$ is satisfiable iff there is an ultimately periodical quasimodel $\boldsymbol{q}$ for $\varphi$ such that $\boldsymbol{q}\left(l_{1}+w\right)=\boldsymbol{q}\left(l_{1}+l_{2}+w\right)$, for every $w \in \mathbb{N}$ and some $l_{1}, l_{2}$ with $l_{1} \leq \sharp(\varphi)$ and $l_{2} \leq k_{\varphi} \cdot \sharp(\varphi)+\sharp(\varphi)$, where $\sharp(\varphi)$ is the number of distinct state candidates for $\varphi$ and $k_{\varphi}$ the number of eventualities in $\varphi$.

Proof. Similar to the proof of [14, Theorem 11.26].
Theorem 9. The satisfiability problem for $\mathcal{Q} \mathcal{T} \mathcal{L}_{\text {krom }}^{1}$ is PSPACE-complete.

Proof. The upper bound follows from Lemma 8 using an algorithm that first guesses $l_{1}$ and $l_{2}$ and then tries to construct an ultimately periodical quasimodel (see [14, Theorem 11.30]). The lower bound follows from PSPACEhardness of $\mathcal{L} \mathcal{T} \mathcal{L}$ (which is a fragment of $\mathcal{Q T} \mathcal{L}_{\text {krom }}^{1}$ ).

\section{TDL-Lite ${ }_{h o r n}$ is EXPSPACE-complete}

Consider the Horn fragment TDL-Lite ${ }_{\text {horn }}$ of

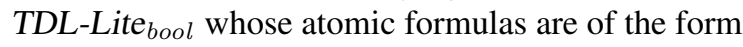

$$
D_{1} \sqcap \cdots \sqcap D_{k} \sqsubseteq D, \quad D\left(a_{i}\right), \quad R\left(a_{i}, a_{j}\right),
$$

where $D, D_{1}, \ldots, D_{k}$ are formed from basic concepts $B$ by means of $\bigcirc$ only as in (20). Again we can apply all temporal operators and the Booleans to formulas. (Note that satisfiability for the underlying DL DL-Lite ${ }_{\text {horn }}$ is P-complete [2].)

Theorem 10. The satisfiability problem for TDL-Lite ${ }_{h o r n}$ is EXPSPACE-complete.

Proof. The upper bound follows from Theorem 4. The lower one is proved by reduction of the $\mathbb{N} \times 2^{n}$ corridor tiling problem that is known to be EXPSPACE-complete (for details see, e.g., $[19,16])$ : given an instance $\left(T, \tau_{0}, n\right)$, where $T$ is a finite set of tile types, $\tau_{0} \in T$ is a tile type, and $n \in \mathbb{N}$ is given in unary, decide whether $T$ tiles the $\mathbb{N} \times 2^{n}$. corridor $\left\{(x, y) \mid x \in \mathbb{N}, 0 \leq y<2^{n}\right\}$ in such a way that $\tau_{0}$ is placed at $(0,0)$ and the top and bottom sides of the corridor are of some fixed colour, say, white. We construct a TDL-Lite ${ }_{\text {horn }}$ formula $\varphi_{T, \tau_{0}, n}$ such that (i) its length is polynomial in $|T|$ and $n$, and (ii) $T$ tiles the $\mathbb{N} \times 2^{n}$ corridor (with $\tau_{0}$ on $(0,0)$ and with white top and bottom sides) iff $\varphi_{T, \tau_{0}, n}$ is satisfiable.

The formula $\varphi_{T, \tau_{0}, n}$ will be constructed in a number of steps. To explain the meaning of its subformulas, let us fix some interpretation $\mathcal{I}$ with some domain $\Delta$.

Let $S_{\tau}$, for $\tau \in T$, be role names and suppose that the following formula holds in $\mathcal{I}$ at 0 :

$$
\square_{F}^{+} \bigvee_{\tau \in T}\left(\top \sqsubseteq \exists S_{\tau}\right) \wedge \bigwedge_{\tau \neq \tau^{\prime}} \square_{F}^{+}\left(\exists S_{\tau} \sqcap \exists S_{\tau^{\prime}} \sqsubseteq \perp\right)
$$

Then there is a uniquely determined sequence $\tau_{0}, \tau_{1}, \ldots$ of tile types such that $\exists S_{\tau_{m}}^{\mathcal{I}(m)}=\Delta$ and $\left(\exists S_{\tau_{m}}^{-}\right)^{\mathcal{I}(m)} \neq \emptyset$, for every $m \in \mathbb{N}$; see Fig. 1 .

Suppose also that the following formulas hold in $\mathcal{I}$ at 0 :

$$
\begin{aligned}
& \bigwedge_{\tau \in T} \square_{F}^{+}\left(\exists S_{\tau}^{-} \sqsubseteq \prod_{j=1}^{n} \bar{Q}_{j} \sqcap \bigcirc N\right), \\
& \bigwedge_{\tau \in T} \square_{F}^{+}\left(\exists S_{\tau}^{-} \sqcap N \sqsubseteq \perp\right), \\
& \square_{F}^{+}(N \sqsubseteq \bigcirc N) .
\end{aligned}
$$




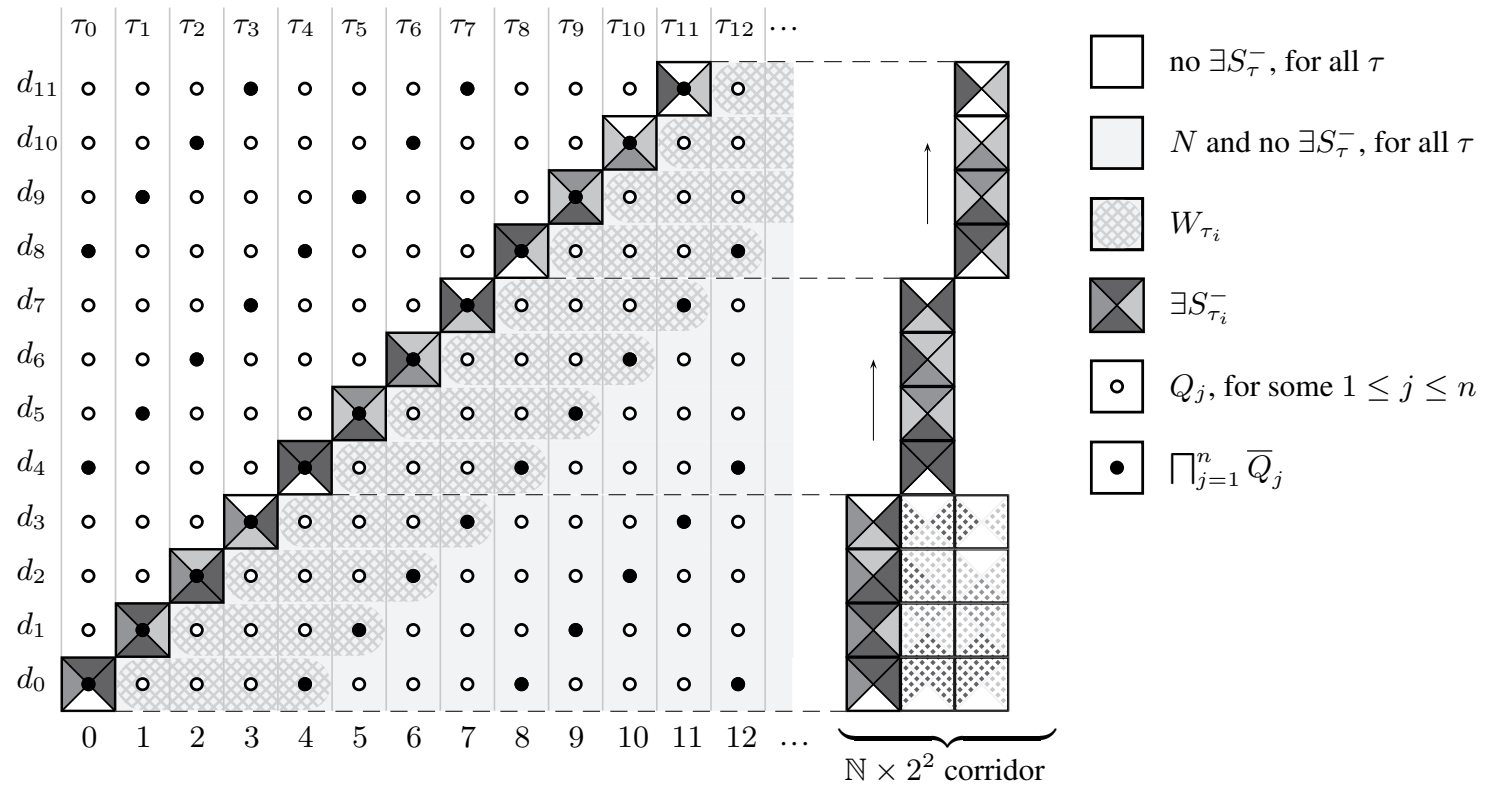

Figure 1. A model $\mathcal{I}$ satisfying $\varphi_{T, \tau_{0}, 2}$.

(Formulas of the form $D_{1} \sqcap \cdots \sqcap D_{k} \sqsubseteq D_{1}^{\prime} \sqcap \cdots \sqcap D_{j}^{\prime}$ are just syntactic sugar.) It follows that at every moment of time $m$ one can select a point $d_{m} \in\left(\exists S_{\tau_{m}}^{-}\right)^{\mathcal{I}(m)}$ such that $d_{m} \in N^{\mathcal{I}(m+1)}$ and $d_{m} \in\left(\bar{Q}_{n} \sqcap \cdots \sqcap \bar{Q}_{1}\right)^{\mathcal{I}(m)}$. The former implies, by (24) and (25), that the $d_{m}$ are all distinct, and the latter will be used to encode a $2^{n}$ counter on $d_{m}$.

The formulas encoding the $2^{n}$ counter on elements of the domain are more or less standard (taking into account that $\bar{Q}_{i}$ stands for the $i$-th bit being 0 and $Q_{i}$ for the $i$-th bit being 1):

$$
\begin{gathered}
\bigwedge_{1 \leq i \leq n} \square_{F}^{+}\left(Q_{i} \sqcap \bar{Q}_{i} \sqsubseteq \perp\right), \\
\bigwedge_{1 \leq j<i \leq n}\left[\square_{F}^{+}\left(\bar{Q}_{i} \sqcap \bar{Q}_{j} \sqcap Q_{j-1} \sqcap \cdots \sqcap Q_{1} \sqsubseteq \square_{F}^{+}\left(Q_{i} \sqcap \bar{Q}_{j} \sqcap Q_{j-1} \sqcap \cdots \sqcap Q_{1} \sqsubseteq \bigcirc Q_{i}\right)\right],\right. \\
\bigwedge_{1 \leq j \leq n}\left[\begin{array}{c}
\square_{F}^{+}\left(\bar{Q}_{j} \sqcap Q_{j-1} \sqcap \cdots \sqcap Q_{1} \sqsubseteq \bigcirc Q_{j}\right) \\
\left.\wedge \square_{F}^{+}\left(Q_{j} \sqcap Q_{j-1} \sqcap \cdots \sqcap Q_{1} \sqsubseteq \bigcirc \bar{Q}_{j}\right)\right] .
\end{array}\right.
\end{gathered}
$$

It follows, in particular, that if the counter is 'initialised' on some $d$, i.e., $d \in\left(\bar{Q}_{n} \sqcap \cdots \sqcap \bar{Q}_{1}\right)^{\mathcal{I}(k)}$, for some $k \in \mathbb{N}$, then

- $d \in\left(\bar{Q}_{n} \sqcap \cdots \sqcap \bar{Q}_{1}\right)^{\mathcal{I}(j)}$ iff $j \equiv k\left(\bmod 2^{n}\right)$;

- there is $1 \leq i \leq n$ such that $d \in Q_{i}^{\mathcal{I}(j)}$ iff $j \not \equiv k$ $\left(\bmod 2^{n}\right)$.

Note also that if $d \notin Q_{i}^{\mathcal{I}(k)} \cup \bar{Q}_{i}^{\mathcal{I}(k)}$, for some $1 \leq i \leq n$ and $k \in \mathbb{N}$, then the counter may not behave properly on $d$.
However, on every $d_{m}$, the counter is initialised at moment $m$ and, therefore, is defined correctly on it.

Let $B$ and the $W_{\tau}$, for $\tau \in T$, be concept names. Then the following formulas ensure correctness of tiling:

$$
\begin{aligned}
& \left(\top \sqsubseteq \exists S_{\tau_{0}}\right) \text {, } \\
& \left(\exists S_{\tau_{0}}^{-} \sqsubseteq B\right) \wedge \square_{F}^{+}(B \sqsubseteq \bigcirc B), \\
& \bigwedge_{\substack{\tau \in T \\
\operatorname{down}(\tau) \neq \text { white }}} \square_{F}^{+}\left(B \sqcap \prod_{j=1}^{n} \bar{Q}_{j} \sqcap \exists S_{\tau} \sqsubseteq \perp\right), \\
& \bigwedge \quad \square_{F}^{+}\left(\exists S_{\tau} \sqcap \bigcirc \exists S_{\tau^{\prime}} \sqsubseteq \perp\right), \\
& \tau, \tau^{\prime} \in T \\
& u p(\tau) \neq \operatorname{down}\left(\tau^{\prime}\right) \\
& \bigwedge_{\substack{\tau, \tau^{\prime} \in T \\
\text { h } \\
(\tau) \neq \operatorname{left}\left(\tau^{\prime}\right)}} \square_{F}^{+}\left(\exists S_{\tau}^{-} \sqsubseteq \bigcirc W_{\tau^{\prime}}\right), \\
& \bigwedge_{\tau \in T} \square_{F}^{+}\left(W_{\tau} \sqcap \prod_{j=1}^{n} \bar{Q}_{j} \sqcap \exists S_{\tau} \sqsubseteq \perp\right), \\
& \bigwedge_{\tau \in T} \bigwedge_{i=1}^{n} \square_{F}^{+}\left(W_{\tau} \sqcap Q_{i} \sqsubseteq \bigcirc W_{\tau}\right) .
\end{aligned}
$$

Indeed, (26) ensures that $\tau_{0}$ is placed at $(0,0)$ and (27) that $d_{0} \in B^{\mathcal{I}(k)}$, for all $k \in \mathbb{N}$. It follows that we have a 'master counter' (distinguished by the concept $B$ ), which is initialised on $d_{0}$ at 0 and has value 0 at every moment of time, when a tile for the bottom row is being selected. Then (28) guarantees that the bottom of the corridor is coloured white. 
By (29), the adjacent colours of tiles in the same column match. It also follows from (29) that the top of the corridor is also white: the $u p$-colour of a tile in the top row matches the down-colour of the tile at the bottom of the next column, which is white by (28). To make the colours of adjacent tiles in the same row match (such tiles are $2^{n}$ moments of time apart) we use the $2^{n}$ counters. Take $d_{m} \in\left(\exists S_{\tau_{m}}^{-}\right)^{\mathcal{I}(m)}$. By (30), $d_{m} \in W_{\tau^{\prime}}^{\mathcal{I}(m+1)}$, for every tile $\tau^{\prime}$ that cannot be put to the right of $\tau_{m}$ in a correct tiling. As the counter is initialised on $d_{m}$ at moment $m$, it has value 1 at $m+1$. Then, by (32), we have $d_{m} \in W_{\tau^{\prime}}^{\mathcal{I}(m+2)}$, for every tile $\tau^{\prime}$ that cannot be put to the right of $\tau_{m}$. The same argument iteratively applies until the moment $m+2^{n}-1$ and therefore, we have $d_{m} \in W_{\tau^{\prime}}^{\mathcal{I}\left(m+2^{n}\right)}$, for every tile $\tau^{\prime}$ that cannot be put to the right of $\tau_{m}$. But then, by (31), no such tile $\tau^{\prime}$ can be selected as $\tau_{m+2^{n}}$.

It follows that if $\varphi_{T, \tau_{0}, n}$ is satisfiable then $T$ tiles the $\mathbb{N} \times 2^{n}$-corridor. The converse implication is clear. Note that $\varphi_{T, \tau_{0}, n}$ does not use any number restriction.

\section{Temporalised $\mathcal{E} \mathcal{L}$ is undecidable}

In contrast to the positive results above we now show that even a rather weak temporalisation $\mathcal{T} \mathcal{L}_{\mathcal{E} \mathcal{L}}$ of $\mathcal{E} \mathcal{L}$ with global roles and GCIs is undecidable. To prove this we do not need ABox assertions. Moreover, $\diamond_{F}$ will be the only temporal concept constructor, and $\square_{F}^{+}$the only operator applied to formulas. Besides, no local roles are required. Formally, $\mathcal{T} \mathcal{L}_{\mathcal{E} \mathcal{L}}$ concepts $C$ are defined as follows:

$$
C::=\top\left|A_{i}\right| C_{1} \sqcap C_{2}\left|\exists T_{i} . C\right| \diamond_{F} C,
$$

where the $T_{i}$ are global role names. A $\mathcal{T} \mathcal{L}_{\mathcal{E} \mathcal{L}}$ GCI is a formula of the form $\square_{F}^{+}\left(C_{1} \sqsubseteq C_{2}\right.$ ) (often written as $C_{1} \sqsubseteq^{*} C_{2}$ ), where $C_{1}, C_{2}$ are $\mathcal{T} \mathcal{L}_{\mathcal{E} \mathcal{L}}$ concepts. Observe that every set of $\mathcal{T} \mathcal{L}_{\mathcal{E} \mathcal{L}}$ GCIs is satisfiable: they are satisfied in the model where all concepts and roles are interpreted by the whole domain at every time point.

In fact, the interesting reasoning problem for $\mathcal{T} \mathcal{L}_{\mathcal{E} \mathcal{L}}$ is whether a GCI is a logical consequence of a set of GCIs. Without the temporal operators, this problem is known to be decidable in polynomial time [5]. We are now going to show that it is undecidable for $\mathcal{T} \mathcal{L}_{\mathcal{E} \mathcal{L}}$.

Theorem 11. It is undecidable whether a $\mathcal{T} \mathcal{L}_{\mathcal{E L}} G C I$ is a consequence of a finite set of $\mathcal{T} \mathcal{L}_{\mathcal{E} \mathcal{L}} G C I$.

Proof. The proof is by reduction of the following version of the undecidable satisfiability problem for temporalised $\mathcal{A L C}$. Define the concepts $C$ of $\mathcal{T} \mathcal{L}_{\mathcal{A L C}}$ as follows:

$$
\begin{aligned}
C::=\top|\perp| A_{i}|\neg C| C_{1} \sqcup C_{2} \mid \\
\\
\quad C_{1} \sqcap C_{2}\left|\exists T_{i} . C\right| \diamond_{F} C .
\end{aligned}
$$

We introduce $\top, \perp$ and $\sqcup$ as primitive connectives because this will be useful in the reduction below. A $\mathcal{T} \mathcal{L}_{\mathcal{A L C}}$ GCI is of the form $\square_{F}^{+}\left(C_{1} \sqsubseteq C_{2}\right)$, where $C_{1}, C_{2}$ are $\mathcal{T} \mathcal{L}_{\mathcal{A L C}}$ concepts. Say that an $\mathcal{A L C}$ concept $C$ is satisfiable relative to a set of GCIs if there exists a model satisfying $C$ and the set of GCIs. The following is proved in [14]:

Theorem 12. Satisfiability of $\mathcal{A L C}$ concepts relative to sets of $\mathcal{T} \mathcal{L}_{\mathcal{A L C}}$ GCIs is undecidable.

Suppose now that a set of $\mathcal{T} \mathcal{L}_{\mathcal{A L C}}$ GCIs and a concept in $\mathcal{A L C}$ are given. First, we perform a number of satisfiability preserving operations.

(a) Ensure that negation $\neg$ occurs in front of concept names only: for every concept $\neg C$ with complex $C$, introduce a fresh concept name $A$, replace $\neg C$ with $\neg A$, and add $A \sqsubseteq^{*} C$ and $C \sqsubseteq^{*} A$ to the set of GCIs. The resulting concept is satisfiable relative to the resulting set of GCIs if the original one was satisfiable relative to the original set of GCIs.

(b) Ensure that $\neg$ does not occur at all in the set of GCIs nor in the concept: for every concept $\neg A$, introduce a fresh concept name $\bar{A}$, replace every occurrence of $\neg A$ with $\bar{A}$, and add $\top \sqsubseteq^{*} A \sqcup \bar{A}$ and $A \sqcap \bar{A} \sqsubseteq^{*} \perp$ to the set of GCIs.

(c) Ensure that disjunction $\sqcup$ does not occur at all in the set of GCIs nor in the concept: first, modulo introduction of new concept names, we may assume that $\sqcup$ does not occur in the concept and that the only occurrences of $\sqcup$ in the set of GCIs are of the form (i) $A \sqcup B \sqsubseteq^{*} C$ and (ii) $C \sqsubseteq^{*} A \sqcup B$, where $A$ and $B$ are concept names and $C$ is disjunction free. Denote the resulting set of GCIs by $\mathcal{T}$ and the concept by $C_{0}$. Now we replace in $\mathcal{T}$ the former kind of GCI with $A \sqsubseteq{ }^{*} C$ and $B \sqsubseteq^{*} C$. The latter one is replaced with four GCIs

$$
\begin{aligned}
& C \sqsubseteq^{*} \exists R .\left(M \sqcap \diamond_{F} X \sqcap \diamond_{F} Y\right), \\
& \exists R .\left(M \sqcap \diamond_{F}\left(X \sqcap \diamond_{F} Y\right)\right) \sqsubseteq^{*} A, \\
& \exists R .\left(M \sqcap \diamond_{F}\left(Y \sqcap \diamond_{F} X\right)\right) \sqsubseteq^{*} A, \\
& \exists R .\left(M \sqcap \diamond_{F}(X \sqcap Y)\right) \sqsubseteq^{*} B,
\end{aligned}
$$

where $R$ is a fresh global role name and $X, Y$ and $M$ are fresh concept names (for each concept inclusion $C \sqsubseteq^{*} A \sqcup$ $B$ ). Denote by $\mathcal{T}^{\prime}$ the the new set of GCIs. Clearly, if $C_{0}$ is satisfiable relative to $\mathcal{T}^{\prime}$, then $C_{0}$ is satisfiable relative to $\mathcal{T}$. Conversely, suppose that $C_{0}$ is satisfiable relative to $\mathcal{T}$. We may assume that the witness interpretation has an infinite domain $\Delta$. Consider a GCI $C \sqsubseteq^{*} A \sqcup B$. Interpret $R$ in such a way that $R^{\mathcal{I}(n)}$ is a forest of infinite outdegree, i.e., $R^{\mathcal{I}(n)}$ is acyclic, for each $w \in \Delta$ there exist infinitely many $w^{\prime} \in \Delta$ such that $\left(w, w^{\prime}\right) \in R^{\mathcal{I}(n)}$, and for each $w^{\prime}$ there exists at most one $w$ with $\left(w, w^{\prime}\right) \in R^{\mathcal{I}(n)}$. Now interpret $M$ by choosing for each $w \in C^{\mathcal{I}(n)}$ exactly one node $w^{\prime} \in M^{\mathcal{I}(n)}$ with $\left(w, w^{\prime}\right) \in R^{\mathcal{I}(n)}$. This can be done in such a way that $M^{\mathcal{I}(n)} \cap M^{\mathcal{I}(m)}=\emptyset$ for $n \neq m$. Finally, 
interpret $X$ and $Y$ as follows: suppose $\left(w, w^{\prime}\right) \in R^{\mathcal{I}(m)}$, $w \in C^{\mathcal{I}(m)}$, and $w^{\prime} \in M^{\mathcal{I}(m)}$, for some $m$. Then $w \in$ $(A \sqcup B)^{\mathcal{I}(m)}$. If $w \in B^{\mathcal{I}(m)}$, then include $w^{\prime}$ in $X^{\mathcal{I}(m+1)}$ and $Y^{\mathcal{I}(m+1)}$. If $w \in A^{\mathcal{I}(m)} \backslash B^{\mathcal{I}(m)}$, then include $w^{\prime}$ in $X^{\mathcal{I}(m+1)}$ and $Y^{\mathcal{I}(m+2)}$. It can be shown that the extended interpretation $\mathcal{I}$ satisfies $\mathcal{T}^{\prime}$ and $C_{0}$.

Observe that $\mathcal{T}^{\prime}$ and the concept $C_{0}$ only contain the operators $\sqcap, \exists, \top, \perp$, and $\diamond_{F}$. We now reduce satisfiability of $C_{0}$ relative to $\mathcal{T}^{\prime}$ to subsumption in $\mathcal{T} \mathcal{L}_{\mathcal{E} \mathcal{L}}$. Introduce a fresh concept name $L$, replace every occurrence of $\perp$ with $L$ and extend $\mathcal{T}^{\prime}$ with $\exists T . L \sqsubseteq^{*} L$, for every role $T$ from $\mathcal{T}^{\prime}$ and $C_{0}$, and $\diamond_{F} L \sqsubseteq^{*} L$. Then $C_{0}$ is satisfiable relative to $\mathcal{T}^{\prime}$ iff $C_{0}^{\prime} \sqsubseteq^{*} L$ does not follow from the new set $\mathcal{T}^{\prime \prime}$ of GCIs, for the new concept $C_{0}^{\prime}$ : clearly, if $C_{0}$ is satisfiable relative to $\mathcal{T}^{\prime}$, then we obtain an interpretation $\mathcal{I}$ satisfying $\mathcal{T}^{\prime \prime}$ in which $L^{\mathcal{I}(0)}=\emptyset$ but $C_{0}^{\mathcal{I}(0)} \neq \emptyset$. Conversely, if $C_{0}$ is not satisfiable relative to $\mathcal{T}^{\prime}$, then for every interpretation $\mathcal{I}$ with $w \in C_{0}^{\prime \mathcal{I}(0)}$, there exists a $w^{\prime}$ reachable from $w$ following a path along global roles $T^{\mathcal{I}(0)}$ (from $\mathcal{T}^{\prime}$ and $\left.C_{0}\right)$ such that $w^{\prime} \in L^{\mathcal{I}(m)}$. But then, by the new GCIs, $w \in L^{\mathcal{I}(0)}$.

\section{Conclusion}

We have shown that temporalisations of various dialects of DL-Lite are decidable with global roles and GCIs, while temporalisations of $\mathcal{E} \mathcal{L}$ are not. The crucial difference between the two languages is the absence of 'qualified' quantification in DL-Lite. As there is no constructor $\exists R . C$ in $D L$-Lite, we can actually encode global roles using temporal constraints on unary predicates. Although we obtain unintended models where roles are not global, the language is too 'weak' to notice this. Note, however, that these decidability results can easily be ruined by role inclusions. We have also seen that, in contrast to qualified quantification, the presence of Boolean operators does not have any impact on the decision problem: although $\mathcal{E} \mathcal{L}$ does not contain $\sqcup$ and $\neg$, its temporal dimension together with GCIs is perfectly capable of reintroducing them.

Acknowledgements. The work on this paper was partially supported by the U.K. EPSRC grants GR/S61966, GR/S63182, GR/S63175, GR/S61973 and Tones, KnowledgeWeb and InterOp projects. We would like to thank the anonymous referees for their comments.

\section{References}

[1] A. Artale. Reasoning on temporal conceptual schemas with dynamic constraints. In Proc. of TIME 2004, pages 79-86. IEEE Computer Society, 2004.

[2] A. Artale, D. Calvanese, R. Kontchakov, and M. Zakharyaschev. DL-Lite in the light of first-order logic. In Proc. of AAAI, 2007.
[3] A. Artale, C.Parent, and S. Spaccapietra. Modeling the evolution of objects in temporal information systems. In Proc. of FoIKS-06, volume 3861 of LNCS, pages 22-42. Springer, 2006.

[4] A. Artale and E. Franconi. Temporal description logics. In M. Fisher, D. Gabbay, and L. Vila, editors, Handbook of Time and Temporal Reasoning in Artificial Intelligence, pages 375-388. 2005.

[5] F. Baader, S. Brandt, and C. Lutz. Pushing the $\mathcal{E} \mathcal{L}$ envelope. In Proc. of IJCAI 2005, pages 364-369, 2005.

[6] F. Baader, R. Küsters, and F. Wolter. Extensions to description logics. In Description Logic Handbook, pages 219-261. Cambridge University Press, 2003.

[7] F. Baader, C. Lutz, and B. Suntisrivaraporn. Is tractable reasoning in extensions of the description logic $\mathcal{E} \mathcal{L}$ useful in practice? In Proc. of the Methods for Modalities Workshop (M4M 2005), 2005.

[8] F. Baader, C. Lutz, and B. Suntisrivaraporn. CEL-a polynomial-time reasoner for life science ontologies. In Proc. of IJCAR'06, volume 4130 of LNAI, pages 287-291. Springer, 2006.

[9] P. Balbiani and J.-F. Condotta. Computational complexity of propositional linear temporal logics based on qualitative spatial or temporal reasoning. In Proc. of FroCoS 2002, volume 2309 of LNCS, pages 162-176. Springer, 2002.

[10] D. Calvanese, G. De Giacomo, D. Lembo, M. Lenzerini, and R. Rosati. DL-Lite: Tractable description logics for ontologies. In Proc. of AAAI 2005, pages 602-607, 2005.

[11] D. Calvanese, G. De Giacomo, D. Lembo, M. Lenzerini, and R. Rosati. Data complexity of query answering in description logics. In Proc. of KR 2006, pages 260-270, 2006.

[12] A. Degtyarev, M. Fisher, and B. Konev. Monodic temporal resolution. ACM Transactions in Computational Logic, 7:108-150, 2006.

[13] D. Gabbay, I. Hodkinson, and M. Reynolds. Temporal Logic: Mathematical Foundations and Computational Aspects, Volume 1. Oxford University Press, 1994.

[14] D. Gabbay, A. Kurucz, F. Wolter, and M. Zakharyaschev. Many-Dimensional Modal Logics: Theory and Applications. Elsevier, 2003.

[15] D. Gabelaia, R. Kontchakov, A. Kurucz, F. Wolter, and M. Zakharyaschev. Combining spatial and temporal logics: expressiveness vs. complexity. JAIR, 23:167-243, 2005.

[16] I. Hodkinson, R. Kontchakov, A. Kurucz, F. Wolter, and M. Zakharyaschev. On the computational complexity of decidable fragments of first-order linear temporal logics. In M. Reynolds and A. Sattar, editors, Proc. of TIME-ICTL 2003, pages 91-98. IEEE Computer Society, 2003.

[17] I. Hodkinson, F. Wolter, and M. Zakharyaschev. Decidable fragments of first-order temporal logics. Annals of Pure and Applied Logic, 106:85-134, 2000.

[18] C. S. Jensen, J. Clifford, R. Elmasri, S. K. Gadia, P. Hayes, and S. Jajodia. The consensus glossary of temporal database concepts. SIGMOD Record, 23(1):52-64, 1994.

[19] P. van Emde Boas. The convenience of tilings. In A. Sorbi, editor, Complexity, Logic and Recursion Theory, volume 187 of Lecture Notes in Pure and Applied Mathematics, pages 331-363. Marcel Dekker Inc., 1997. 